Journal of Luminescence $18 / 19$ (1979) 51-56

(C) North-Holland Publishing Company

\title{
INTERMOLECULAR RESONANCES, OPTICAL DEPHASING AND RELAXATIONS IN A CRYSTALLINE COMPLEX*
}

\author{
P.N. PRASAD $\dagger$, K.S. LAW and J.C. BELLOWS \\ Department of Chemistry, State University of New York at Buffalo, \\ Buffalo, New York 14214, USA \\ and
}

\section{A.H. FRANCIS}

Department of Chemistry, University of Michigan, Ann Arbor, Michigan 48104, USA

\begin{abstract}
Singlet-triplet transition is investigated for 1,4-dibromonaphthalene: octafluoronaphthalene complex where the triplet states of the two components are in near resonance. Photo excitation, $T_{1} \leftarrow S_{0}$ absorption and phosphorescence spectra reveal weak intercomponent quasi-resonance interactions, but rapid intercomponent relaxation. $T_{1} \leftarrow S_{0}$ absorption lines are considerably broader than that in neat 1,4-dibromonaphthalene.
\end{abstract}

A crystalline complex consists of a rich manifold of energy states in which the study of optical dephasing and relaxation of excited states can provide valuable insight into the nature of these processes in condensed media. The work done in our laboratory shows that octafluoronaphthalene is extremely versatile in forming $1: 1$ complexes with aromatic hydrocarbons and their derivatives [1]. These complexes are primarily stabilized by Van der Waals interactions, although in favorable cases other kinds of interactions may provide additional stabilization [1].

By a judicious choice of component 1 the triplet states of the two components can be brought into near resonance. The objective of this work was to investigate the manifestations of such intercomponent resonances on relaxation and optical dephasing processes. For this reason the present study was focused on complexes between halonaphthalenes and octafluoronaphthalene. At this point only the complex 1,4-dibromonaphthalene: octafluoronaphthalene has been investigated.

This paper presents results on the triplet states in 1,4-dibromonaphthalene: octafluoronaphthalene (DBN:OFN) to derive information on the intercom-

* Supported in part by NSF Grant No. DMR75-02628 and in part by the Petroleum Research Fund Administered by the American Chemical Society.

$\dagger$ Alfred P. Sloan Fellow. 
ponent resonance interaction, relaxation and optical dephasing in this crystalline complex.

First, we discuss the case of intercomponent resonance interaction. Fig. I shows the photo-excitation spectra at $2 \mathrm{~K}$ of the complex and the neat 1,4dibromonaphthalene in the region of $T_{1} \leftarrow S_{0}$ transition, in each monitoring all line emission. Except for the doubling observed in neat 1,4-dibromonaphthalene$h_{6}\left(D B N-h_{6}\right)$ due to two molecules per asymmetric unit, there is a one to one correspondence between the spectra of the DBN:OFN complex and that of the neat DBN. This correlation shows that the photo-excitation spectra maps the triplet absorption of DBN in the environment of the complex. The deuteration shift observed in going from DBN- $h_{6}:$ OFN to DBN-d $d_{6}: O F N$ is $\approx 65 \mathrm{~cm}^{-1}$, which

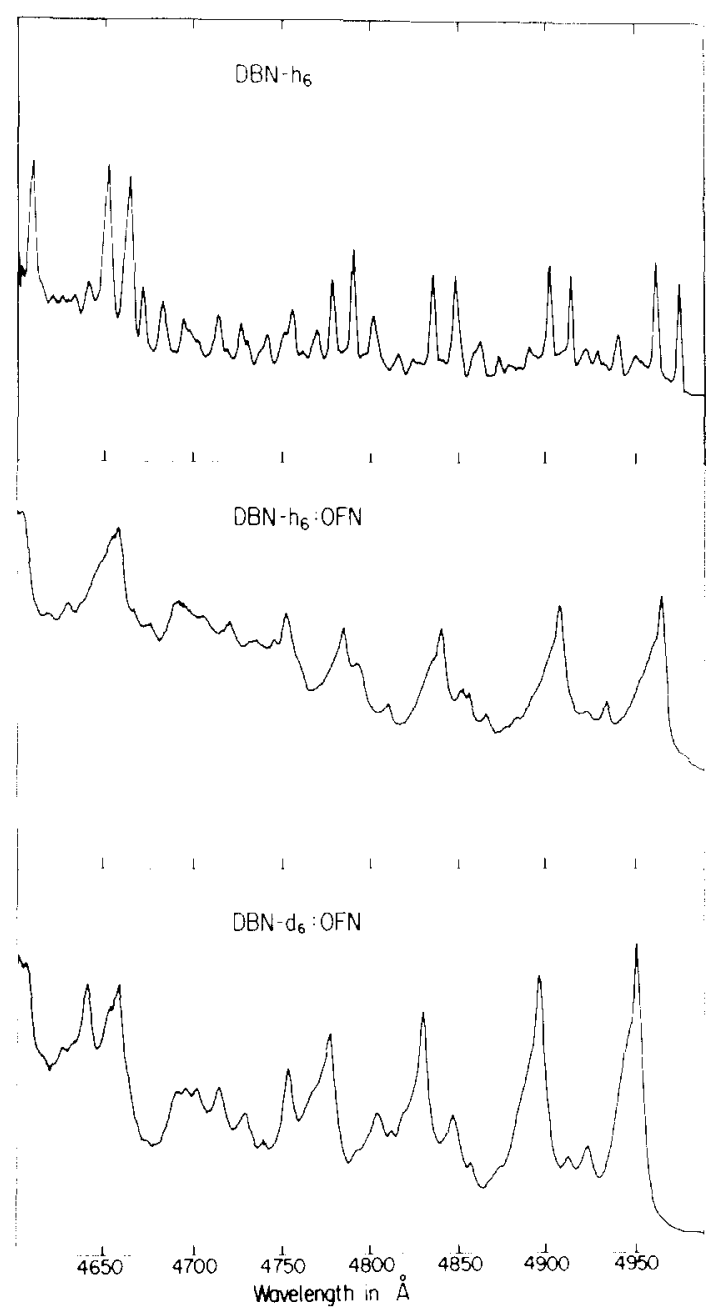

Fig. 1. Photoexcitation spectra at $2 \mathrm{~K}$ of 1,4-dibromonaphthalene: octafluoronaphthalene complex and neat 1.4-dibromonaphthalene in the region of $T_{1} \leftarrow S_{0}$ transition. 
is the same as that observed for neat DBN [2]. This result indicates that the quasi-resonance interaction is small. The exact correlation between the DBN$\mathrm{h}_{6}$ : OFN and neat DBN photo-excitation spectra indicates the absence of a two-particle transition involving the triplet state of DBN and a vibration of OFN. The $T_{1} \leftarrow S_{0}$ absorption and $T_{1} \rightarrow S_{0}$ phosphorescence spectra also provide no evidence for a two-particle transition. These two spectra for the DBN$h_{6}$ : OFN complex at $2 \mathrm{~K}$ are shown in fig. 2. Both $T_{1} \leftarrow \mathrm{S}_{0}$ absorption and $T_{1} \rightarrow S_{0}$ phosphorescence spectra are characteristic of DBN- $h_{6}$.

There is an $80 \mathrm{~cm}^{-1}$ gap between the absorption and the emission origin. The life time of the phosphorescence emission is essentially the same as observed in neat DBN- $h_{6}$. However, the criteria of using two-particle transitions and lifetimes are not sensitive enough for the complex DBN:OFN. The reason is that the $T_{1} \leftarrow S_{0}$ transition in DBN is much stronger than that in OFN. Nevertheless, considering that no quasi-resonance shift is observed as well as the facts that there is no evidence for a two-particle transition and the lifetime of

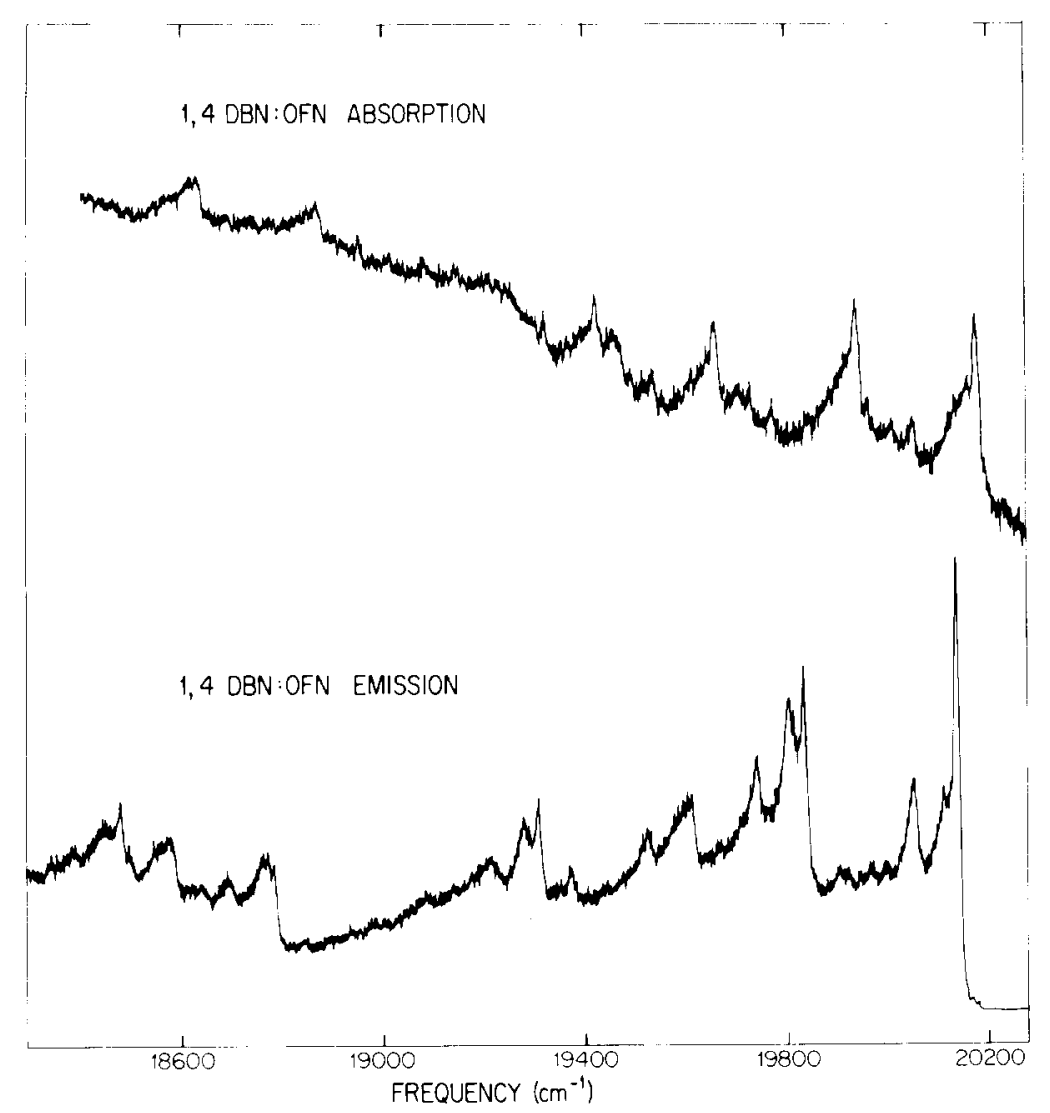

Fig. 2. Absorption and emission spectra of 1,4-dibromonaphthalene- $\mathrm{h}_{6}$ : octafluoronaphthalene complex at $20 \mathrm{~K}$. 
phosphorescence is essentially the same, we conclude that the triplet states of DBN and OFN are only weakly interacting even though their separation is within $\sim 200 \mathrm{~cm}^{-1}$.

Next we discuss the case of relaxation. As the phosphorescence spectra consists of emission only from DBN, there is a quenching of OFN phosphorescence by DBN. The complexes of OFN with naphthalene and durene as well as neat OFN show strong OFN phosphorescence by a rapid inter-system crossing. The life-time of the OFN emission in these systems is on the order of $\approx 0.4 \mathrm{~s}$. On the other hand, the life-time of DBN emission in DBN: complex is on the order of milliseconds. Raising the temperature from $2 \mathrm{~K}$ to $25 \mathrm{~K}$ does not have any significant change on the emission spectra of the complex. As we saw earlier, the phosphorescence photo excitation spectra of the complex in the triplet region maps that $T_{1} \leftarrow \mathrm{S}_{0}$ absorption of $\mathrm{DBN}$ but this is probably due to the fact that the oscillator strength of $D B N T_{1} \leftarrow S_{0}$ is much greater than that for $\mathrm{T}_{1} \leftarrow \mathrm{S}_{0}$ transition of OFN. However, the photo excitation spectra of DBN$h_{6}$ : OFN in the region of $S_{1} \leftarrow S_{0}$ transitions reveals additional bands which we assign to OFN. Thus, a relaxation from $S_{1}$ of OFN to $T_{1}$ of DBN occurs which quenches the emission from $T_{1}$ of $\mathrm{OFN}$ even though the intercomponent quasi-resonance interaction is weak. However, more experiments are needed to establish if the transfer occurs as $S_{1}^{\mathrm{OFN}} \leadsto T_{1}^{\mathrm{OFN}} \leadsto T_{1}^{\mathrm{DBN}}$ or $S_{1}^{\mathrm{OFN}} \leadsto \mathrm{S}_{1}^{\mathrm{DBN}} \leadsto T_{1}^{\mathrm{DBN}}$.

The study of optical dephasing in these crystalline complexes can provide valuable insight into the nature of these processes in multi-level systems. Our study of the dephasing process utilizes the line width and line shape of the $T_{1} \leftarrow S_{0}$ absorption. Fig. 3 shows the spectrum in the region of the $T_{1} \leftarrow S_{0}$ absorption origin at a greater resolution for both $D B N-h_{6}$ and $D B N-h_{6}:$ OFN crystalline complex at $2 \mathrm{~K}$. The peak at $\approx 20 \mathrm{~cm}^{-1}$ from the origin in the DBN- $h_{6}:$ OFN is due to phonon side band. The inference is derived from a temperature dependence study. The relative intensity of this side band increases with an increase in temperature. On the other hand, in case of neat DBN- $\mathrm{h}_{6}$ no phonon side band is observed [3], which we had previously attributed to exciton delocalization that reduces the local lattice relaxation responsible for the phonon side band [3]. Thus, there is an enhanced phonon coupling due to local lattice relaxation arising, possibly, from exciton localization. The line width of the transition in DBN : OFN complex is about an order of magnitude greater than that observed in neat DBN. The line broadening is not a homogeneous broadening. This broadening is possibly due to disorder. The crystal structure of this complex has not been determined. A possible source of such a large disorder is the random orientation of bromine groups if $\mathrm{DBN}$ occupies a centrosymmetric site. The line shape appears to be close to a Gaussian curve. Both the line shape and the line width remain virtually unchanged up to $20 \mathrm{~K}$, at which point the growing phonon sideband overlaps with the zero-phonon transition. In order to derive the homogeneous line width and its temperature dependence we plan to conduct hole burning experiments.

Also we are planning to investigate the complexes of 1-bromonaphthalene and 2,3-dibromonaphthalene with octafluoronaphthalene. These complexes would provide a unique opportunity to study the dynamics of energy states for the case 


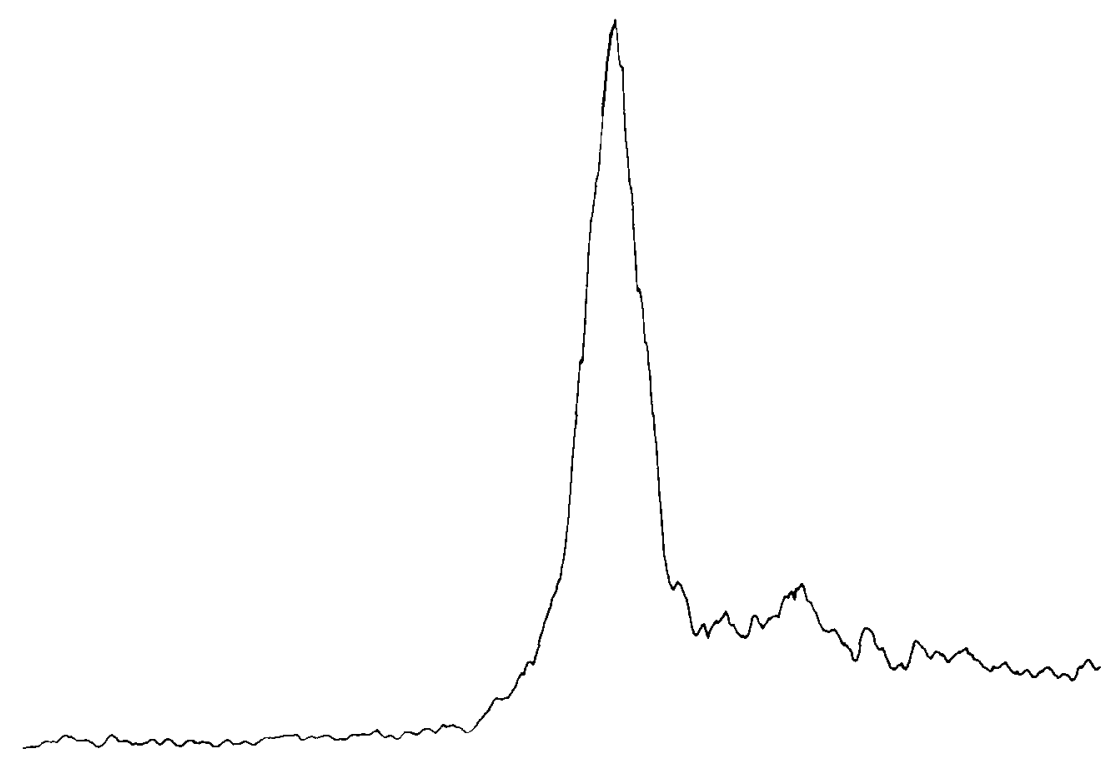

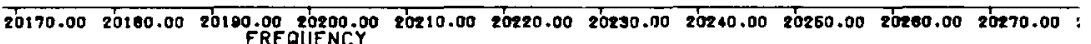

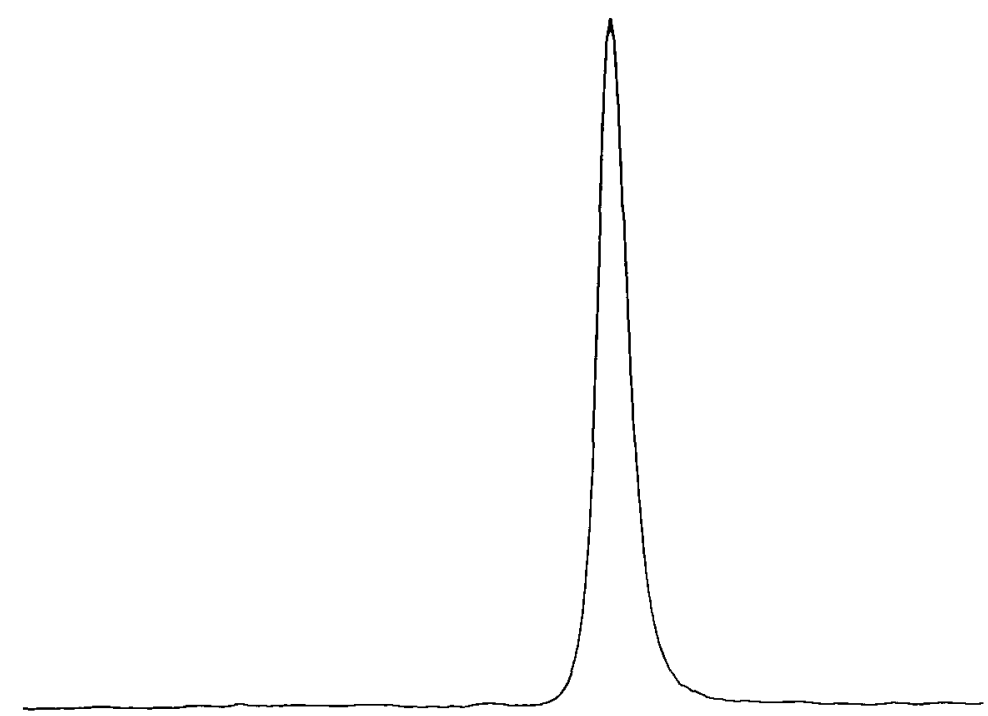

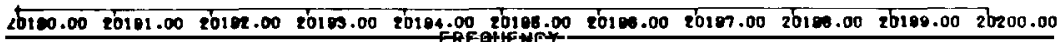

Fig. 3. $T_{1} \leftarrow S_{0}$ absorption spectra near the origin in both neat 1,4-bromonaphthalene (bottom) and 1,4-dibromonaphthalene: octafluoronaphthalene (top) at $2 \mathrm{~K}$. 
where a strongly active triplet of halonaphthalene is embedded in a triplet energy manifold of octafluoronaphthalene with a considerably lower oscillator strength.

\section{References}

[1] F.P. Chen and P.N. Prasad, to be published.

[2] R.M. Hochstrasser and J.D. Whiteman, J. Chem. Phys. 56 (1972) 5945.

[3] R.M. Hochstrasser and P.N. Prasad, J. Chem. Phys. 56 (1972) 2814 